\title{
CONTROL OF NON-LINEAR SYSTEM USING BACKSTEPPING
}

\author{
Anamika Vyas Ojha', Achala Khandelwal ${ }^{2}$ \\ ${ }^{I}$ Asst Prof, Department of Electronics and Communication Engineering, Acropolis Institute of Technology \& \\ Research, Madhya Pradesh, India \\ ${ }^{2}$ Asst Prof, Department of Electrical Engineering, Shri Vaishnav Institute of Technology \& Science, Madhya Pradesh, \\ India
}

\begin{abstract}
The defining attribute of a servomechanism is that the controlled output of a mechanism is automatically compared with the controlling input. The difference between the settings or positions of the output and the input is called the error signal, which acts to bring the output to its desired value.The paper work shows the application of backstepping technique to stabilize a nonlinear dynamical system. Any nonlinear dynamical system can be stabilized only by means of Lyapunov function approach. In this approach backstepping technique is invariably adopted. In case of normal backstepping technique stabilizing function is designed at every step of dynamics starting from the output towards input. The Backstepping approach provides a recursive method for stabilizing the origin of a system in strict-feedback form. The controller contains the terms of system state variables, reference input \& its derivatives, and error. The basic aim of the controller is to settle down error and its derivatives to zero. The controller is designed through Lyapunov based function so as to render strong closed loop stability. In the theory of control systems, Lyapunov functions are scalar functions that may be used to prove the stability of equilibrium of a control system.
\end{abstract}

Keywords: Lyapunov function, backstepping, nonlinear system, stability, derivative $* * *$

\section{INTRODUCTION}

Prior moving towards the projected technique we must be acquainted with some common terminology. We must have a clear comprehension of control system. A control system is a seamless interconnection of components associated in such a manner as to command and regulates itself or some other system. A control system that manages the behaviour of another system is called a Controller. On the foundation of number of input-output a system can either be elucidated as a MIMO system, with multiple inputs and outputs, therefore needing more than one controller. And SISO system, comprising of a single input and single output, hence having only a single controller. Depending on the set-up of the system, adjusting the system's input variable will influence the operating parameter, otherwise known as the controlled output variable. On receiving the error signal that marks the discrepancy between the desired output and the actual output, the controller will then stab to regulate controlled output behavior.

The system may be linear or nonlinear that is being controlled by a controller. In the same way the methods are different for designing a controller for linear and non-linear systems. A linear system is one which satisfies the properties of superposition and scaling or homogeneity. One more aspect of a linear system is that its output is directly proportional to its input. Also these systems have one equilibrium point at the origin. A non-linear system is one which does not obey any of the above properties. Each system must assure the stability for closed loop behaviour. Stability needs to be precisely defined for nonlinear systems. For linear systems this can be obtained by directly placing the poles.

\subsection{Where Do Nonlinearities come from?}

Many of physical quantities, such as a vehicle's velocity, or electrical signals, have an upper bound. When that upper bound is reached, linearity is lost. The differential equations governing some systems, such as some thermal, fluidic, or biological systems, are nonlinear in nature. It is therefore advantageous to consider the nonlinearities directly while analyzing and designing controllers for such systems. Mechanical systems may be designed with backlash - this is so a very small signal will produce no output (for example, in gearboxes). In addition, many mechanical systems are subject to nonlinear friction. Relays, which are part of many practical control systems, are inherently nonlinear. Finally, ferromagnetic cores in electrical machines and transformers are often described with nonlinear magnetization curves and equations.

\section{METHODOLOGY}

\subsection{Backstepping}

Non-linear systems use specific theories and methods to ensure stability without regard to the inner dynamics of the system. Backstepping and Integrator backstepping control are few of the methods. Backstepping is a technique developed circa 1990 by Petar V. Kokotovic and others for designing stabilizing controls for a special class of nonlinear dynamical systems. These systems are built from subsystems that radiate out from an irreducible subsystem that can be stabilized using some other method. Because of this recursive structure, the designer can start the design process at the known-stable system and "back out" new controllers that progressively stabilize each outer subsystem. The process terminates when the final external control is reached. Hence, this process is known as Backstepping 


\subsection{Controller Design of Second Order Nonlinear}

\section{System}

An example to illustrate Backstepping Control applied on a second order single input nonlinear system. The system is given by equations

$$
\left.\begin{array}{l}
\dot{x}_{1}=x_{2}+\theta_{1} \varphi_{1}\left(x_{1}\right) \\
\dot{x}_{2}=\theta_{2} \varphi_{2}\left(x_{1}, x_{2}\right)+b u
\end{array}\right\}
$$

Here we consider $b$ a constant. Let the first error be

$$
z_{1}=y_{R}-x_{1}
$$

Taking derivative

$$
\begin{gathered}
\dot{z}_{1}=\dot{y}_{R}-\dot{x}_{1}=\dot{y}_{R}-x_{2}-\theta_{1} \varphi_{1} \\
\dot{z}_{1}=y_{R}-x_{2}-\theta_{1} \varphi_{1}
\end{gathered}
$$

The Lyapunov function is selected as

$$
V_{1}=\frac{1}{2} z_{1}^{2}
$$

Then,

$$
\dot{V}_{1}=z_{1} \dot{z}_{1}=z_{1}\left(\dot{y}_{R}-x_{2}-\theta_{1} \varphi_{1}\right)
$$

From equation above, we get

$$
\dot{V}_{1}=z_{1}\left(\dot{y}_{R}-x_{2}-\theta_{1} \varphi_{1}\right)
$$

Let the second error be

$$
z_{2}=\alpha-x_{2}
$$

Taking derivative

$$
\dot{z}_{2}=\dot{\alpha}-\dot{x}_{2}=\dot{\alpha}-\theta_{2} \varphi_{2}-b u
$$

Then

$$
\dot{V}_{1}=z_{1}\left(\dot{y}_{R}-\alpha+z_{2}-\theta_{1} \varphi_{1}\right)
$$

To make $\dot{V}_{1}<<0$, choose $\alpha$ such that

$$
\alpha=\dot{y}_{R}-\theta_{1} \varphi_{1}+c_{1} z_{1}
$$

where $\quad c_{1}>0$
Then

$$
\dot{V}_{1}=-c_{1} z_{1}^{2}+z_{1} z_{2}
$$

Therefore

$$
\dot{z}_{1}=-c_{1} z_{1}+z_{2}
$$

Taking another Lyapunov function

$$
V_{2}=V_{1}+\frac{1}{2} z_{2}^{2}
$$

Then,

$$
\begin{aligned}
\dot{V}_{2}=\dot{V}_{1}+z_{2} \dot{z}_{2} & =-c_{1} z_{1}^{2}+z_{1} z_{2}+z_{2} \dot{z}_{2} \\
\dot{z}_{2} & =\left(\dot{\alpha}-\theta_{2} \varphi_{2}-b u\right)
\end{aligned}
$$

From equation (1), we get

$$
\dot{V}_{2}=-c_{1} z_{1}^{2}+z_{2}\left(z_{1}+\dot{\alpha}-\theta_{2} \varphi_{2}-b u\right)
$$

After solving we get $\dot{\alpha}$ as

$$
\dot{\alpha}=\ddot{y}_{R}-\theta_{1} \frac{\partial \varphi_{1}}{\partial x_{1}} \dot{y}_{R}+\left(\theta_{1} \frac{\partial \varphi_{1}}{\partial x_{1}}+c_{1}\right)\left(-c_{1} z_{1}+z_{2}\right)
$$

Then,

$\dot{V}_{2}=-c_{1} z_{1}^{2}+z_{2}\left[z_{1}+\ddot{y}_{R}-\theta_{1} \frac{\partial \varphi_{1}}{\partial x_{1}} \dot{y}_{R}+\left(\theta_{1} \frac{\partial \varphi_{1}}{\partial x_{1}}+c_{1}\right)\left(-c_{1} z_{1}+z_{2}\right)-\theta_{2} \varphi_{2}-b u\right]$

Therefore the final control input law $u$ is given by

$$
u=\frac{1}{b}\left[z_{1}+\dot{\alpha}+5 x_{1}^{2} x_{2}^{2}+c_{2} z_{2}\right]
$$

The controller is simulated on MATLAB Simulink Version 7.5 (R2009b) software. The simulation is shown by different block diagram which consists of simulation of system equations as well as controller.

$$
u=\frac{1}{b}\left[z_{1}+\ddot{y}_{R}-\theta_{1} \frac{\partial \varphi_{1}}{\partial x_{1}} \dot{y}_{R}+\left(\theta_{1} \frac{\partial \varphi_{1}}{\partial x_{1}}+c_{1}\right)\left(-c_{1} z_{1}+z_{2}\right)-\theta_{2} \phi_{2}+c_{2} z_{2}\right]
$$

Consider

$$
\theta_{1}=2, \varphi_{1}=x_{1}^{3}, \theta_{2}=-5, \varphi_{2}=x_{1}^{2} x_{2}^{2}, b=2
$$


In Fig1, first block is the reference block of simulation of a second order nonlinear system. It consists of different blocks as $\mathrm{yr}$ and its derivative block with unit step signal as its input, next block is a controller system where the above given control law is designed, than block diagram of second order nonlinear system block which is to be stabilized and a recording system block which stores the result when we run the simulated system. The output of the controller is stabilizing function for the second order nonlinear system. A step signal of unity magnitude is the reference input for the system given to yr and derivative block. The recording system is to display the simulation results.

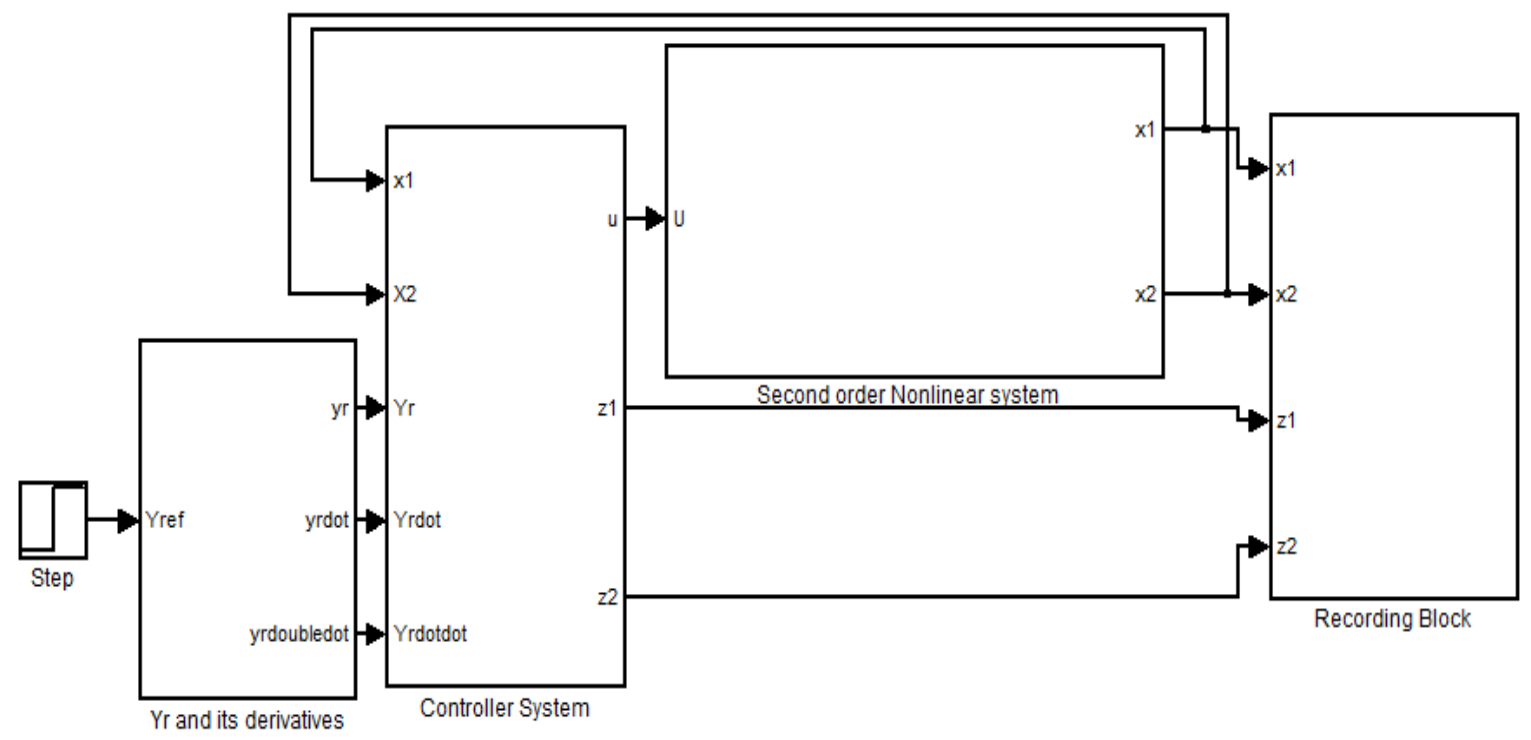

Fig -1: Simulation block diagram of second order nonlinear system

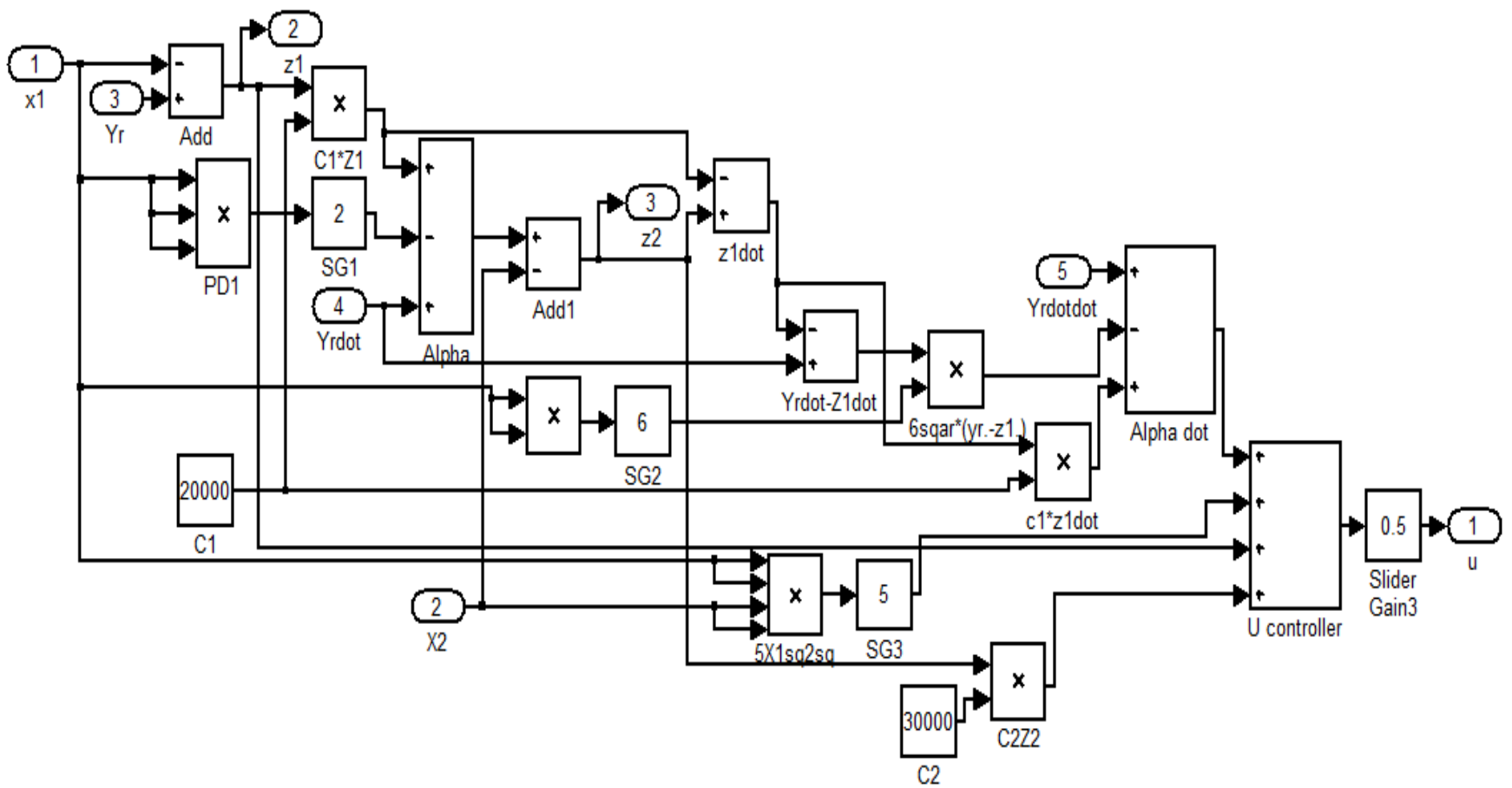

Fig -2: Controller system block

\section{RESULTS}

Fig. 3 shows the graph of state variable $\mathrm{x} 1$ with respect to time t, to make error $\mathrm{z} 1$ zero, state vector $\mathrm{x} 1$ must follow reference input yr. In Fig. 3 the initial value of $x 1$ is zero but within fraction of a second it become unity. The final value of $\mathrm{x} 1$ is one show that state $\mathrm{x} 1$ is following the reference input yr that is unit step signal. Fig. 4 shows graph of state vector $\mathrm{x} 2$ with respect to time $\mathrm{t}$, to make error $\mathrm{z} 2$ to be zero $\mathrm{x} 2$ must follow alpha, which settle down to -2 . The initial value of $\mathrm{x} 2$ is zero, but within fraction of a second it becomes -2 . The final value of $x 2$ in the graph is -2 which the desired value as it is following 


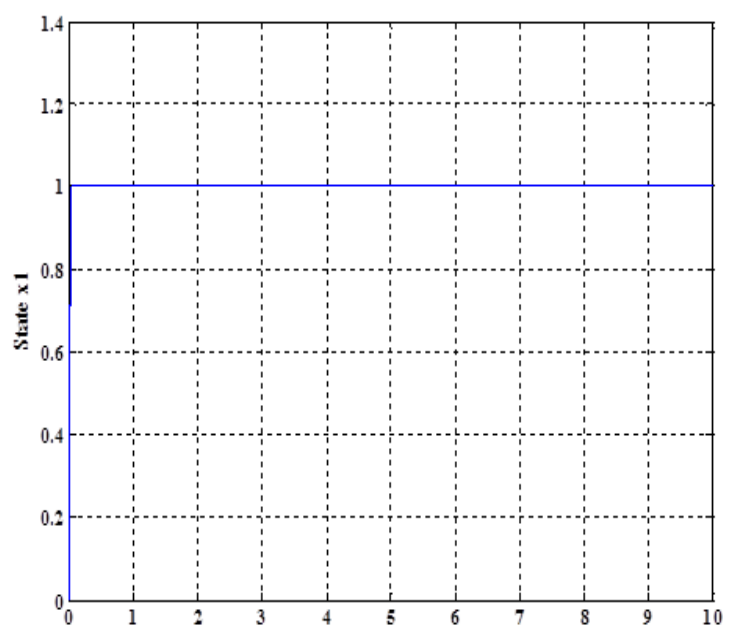

Fig -3: State Response of State Vector x1

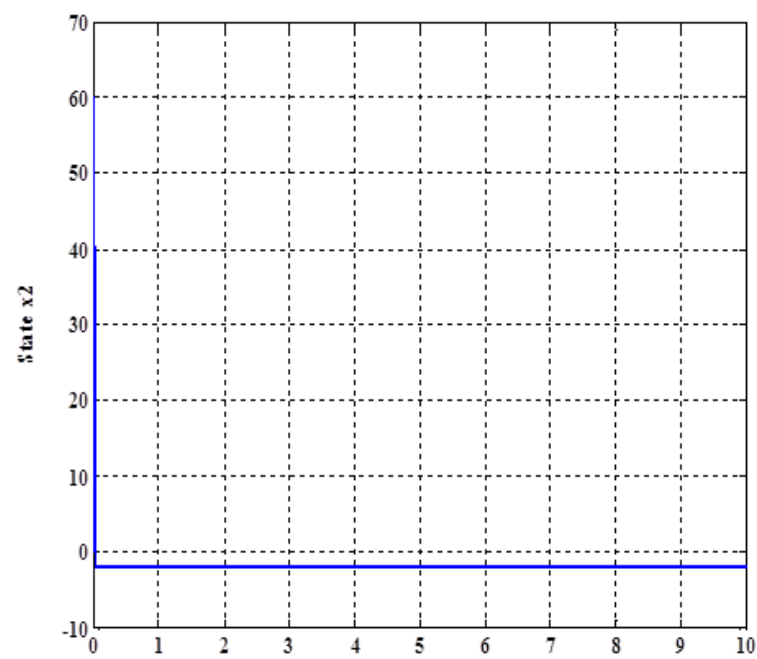

Fig -4: State Response of State Vector x2

Fig.4 and Fig.5 shows the graphs of errors $\mathrm{z} 1$ and $\mathrm{z} 2$ respectively. All this errors must die out to zero to get desired result. The initial and final values of these errors are shown in figure. The first two errors z1 and z2 correspond to system state vectors $\mathrm{x} 1$ and $\mathrm{x} 2$. Final values of errors and are practically zero so we can expect system to be stable.

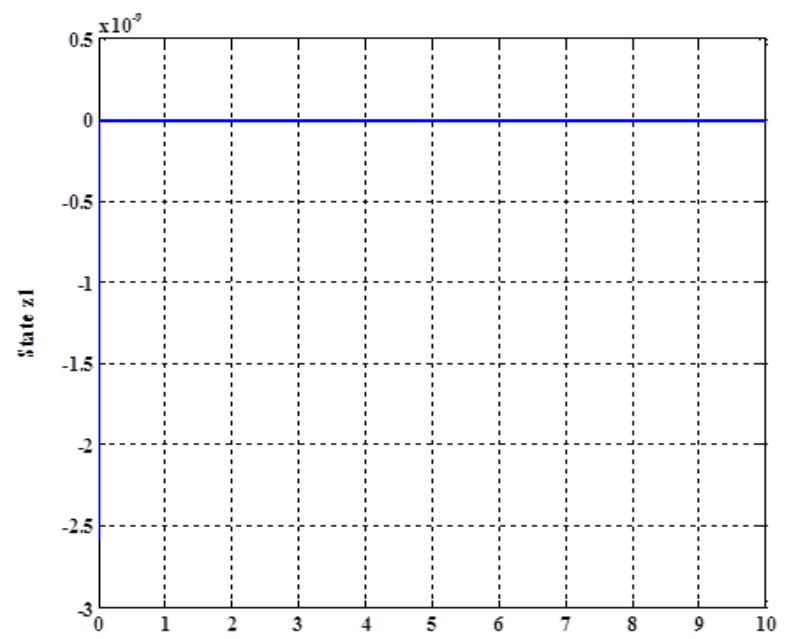

Fig -5: State Response of error z1

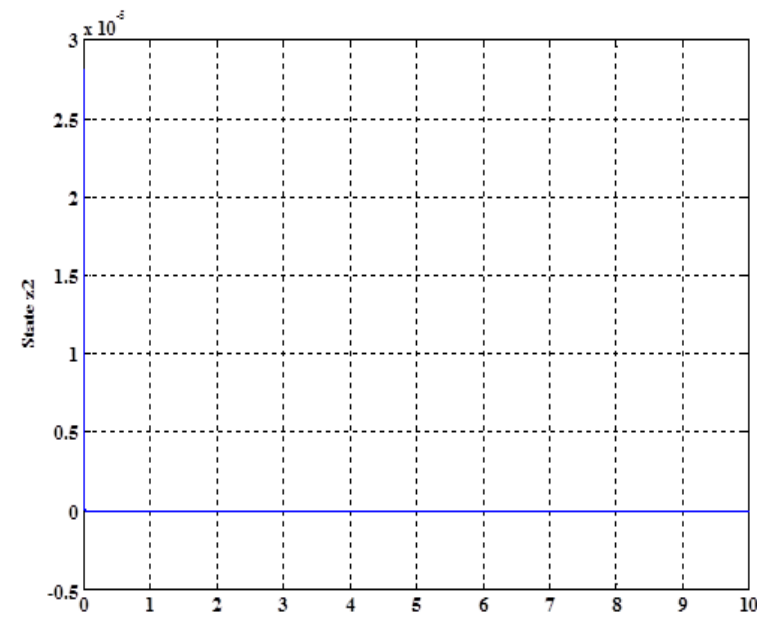

Fig -6: State Response of error z2

\section{CONCLUSION}

The results shown above are of second order and systems. From the results it can be seen that after applying backstepping control technique the stability of the system improved and the performance with respect to time is reliable. We can also conclude that for multiple input systems it is more easier to control the system with this technique So we get a flexibility in designing the control input law during simulation. Backstepping controller recursively uses Lyapunov functions in each integrator level to cancel the nonlinear terms which ensures asymptotic stability.

\section{REFERENCES}

[1]. R A Freeman and P V Kokotović, "Backstepping design of robust controllers for a class of nonlinear systems," Proceedings of the IFAC Nonlinear Control Systems Design Symposium, Bordeanx, France, June 1992, pp. 307-312.

[2]. H K Khalil, Nonlinear Systems, New York: Macmillan, 1992.

[3]. D M Dawson, J J Carroll and M Schneider, "Integrator backstepping control of a brushed DC motor turning a robotic load," IEEE Transactions on Control System Technology, Vol. 2, pp. 233-244, 1994.

[4]. Z P Jiang and J B Pomet, "Combining backstepping and time varying techniques for a new set of adaptive controllers," Proceedings of the $33^{\text {rd }}$ IEEE conference on Decision and Control, Lake Buena Vista, FL, December 1994, pp. 2207-2212.

[5]. Miroslav Krstić, Ioannis Kanellakopoulos and Petar Kokotović, "Nonlinear and Adaptive Control Design", A Wiley-Interscience Publication 1995, John Wiley \& sons, Inc.

[6]. R A Freeman and P V Kokotović, Robust Nonlinear Control Design. Boston, M A Birkhauser, 1996. 


\section{BIOGRAPHIES}

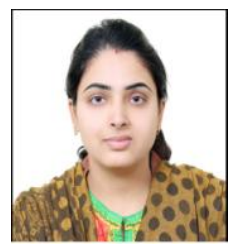

Mrs Anamika Vyas Ojha, Asst Prof,

Acropolis Institute of Technology and

Research, Indore ,Madhya Pradesh

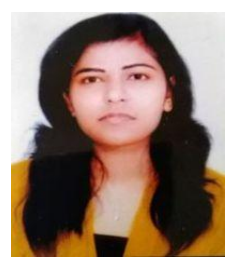

Ms Achala Khandelwal, Asst Prof, Shri

Vaishnav Institute of Technology and

Science Indore, Madhya Pradesh 